\title{
Surface roughness effects on the solar reflectance of cool asphalt shingles
}

\author{
Paul Berdahl and Hashem Akbari \\ Heat Island Group \\ Environmental Energy Technologies Division \\ Lawrence Berkeley National Laboratory \\ Berkeley, CA 94720 \\ and \\ Jeffry Jacobs and Frank Klink \\ Industrial Mineral Products Division \\ 3M Company \\ St. Paul, MN 55144
}

\begin{abstract}
We analyze the solar reflectance of asphalt roofing shingles that are covered with pigmented mineral roofing granules. The reflecting surface is rough, with a total area approximately twice the nominal area. We introduce a simple analytical model that relates the "micro-reflectance" of a small surface region to the "macro-reflectance" of the shingle. This model uses a mean field approximation to account for multiple scattering effects. The model is then used to compute the reflectance of shingles with a mixture of different colored granules, when the reflectances of the corresponding mono-color shingles are known. Simple linear averaging works well, with small corrections to linear averaging derived for highly reflective materials.

Reflective base granules and reflective surface coatings aid achievement of high solar reflectance. Other factors that influence the solar reflectance are the size distribution of the granules, coverage of the asphalt substrate, and orientation of the granules as affected by rollers during fabrication.
\end{abstract}

\section{Introduction}

In warm climates elevated roof temperatures caused by the absorption of solar radiation are undesirable. High roof temperatures increase air conditioning energy use and/or reduce occupant comfort. Modern cool roofing materials are formulated by designing materials with low solar absorptance subject to the constraints of desired visible color and texture. Thus, pigments with light, medium, and dark colors are employed which either have high near-infrared (NIR) reflectance ( 700 to $2500 \mathrm{~nm}$ ), or which are transparent in the NIR and used over a reflective substrate [1-3].

Of the various common roofing types (metal, tile, wood, etc.), formulation of cool asphalt shingles is particularly challenging. The inherently high surface roughness reduces reflectance below what would be achieved with a similar, but smooth surface. The need to achieve low cost also limits the number and type of pigmented coatings that can be applied to the granules. 
Asphalt shingles are fabricated by pressing pigmented mineral granules into an asphaltimpregnated fiberglass mat. Granule sizes vary from about 0.4 to $2 \mathrm{~mm}$; a distribution of sizes aids good coverage. The granules cover the sheet so completely that only a small fraction of the asphalt $(<5 \%)$ is exposed. Thus solar absorptance by the black asphalt is not very important for a well-fabricated shingle. The granule reflectance, before and after application of the thinpigmented coating, is very important. The coating is a porous glass-like film, composed of silica, sodium and/or potassium oxide, and clay. The coating thickness is usually in the range of 5 to 15 micrometers.

In the analysis of the solar reflectance of shingles, as a matter of principle, one should treat each wavelength as independent. Then, having computed the reflectance at each wavelength, one can compute the weighted average solar reflectance, using a standard solar spectrum as a weighting function. In the theoretical models to be described in this paper, then, the reflectance $R$ is to be understood to be the spectral reflectance. In some special cases, the need for spectral averaging can either be approximately or accurately ignored. For example a mixture of gray and black granules can be accurately treated without spectral averaging if the gray and black reflectances are nearly independent of wavelength.

Figure 1 shows photographic close-ups of three experimental samples with light brown granules. Figure 1 (a) shows a section of sample very similar to a manufactured shingle except that it is monocolor; manufactured shingles typically use blends of several colors. The samples in Figs. 1 (b) and (c) were fabricated by hand in a laboratory using black and white acrylic paint as adhesive (instead of asphalt) on a metal substrate. Granules were dropped onto the paint, and then pressed in with a glass plate. It can be seen that the granules of Figs. 1 (b) and (c) do not lie as flat as those of Fig. 1(a). The rolling process used to make sample (a) produced a smoother texture. The solar reflectances (measured with a Solar Spectrum Reflectometer as discussed in Sec. 4) corresponding to Figs. 1 (a), (b), and (c) are 0.246, 0.209, and 0.232, respectively. If each of these samples were fabricated using a black adhesive, we might attribute the reflectance variations as due to coverage variations. However, even the use of a white adhesive did not lead to the highest reflectance (a smooth white coating has a solar reflectance of about 0.8 ).

Therefore, we attribute much of the reflectance variation to the orientation of the granules on the substrate. Later on in this paper, we will use our theoretical model, together with the reflectances corresponding to Figs. 1 (b) and (c), to obtain a rough idea how much the dark adhesive does affect the shingle reflectance.

\section{Reflectance of a rough surface: mean field approximation}

We adopt notation as follows. The macroscopic reflectance of a surface such as a shingle is denoted $R$. The corresponding absorptance is $A$, which is just $1-R$ for an opaque surface. The "microscopic" reflectance is denoted $r$. This is the reflectance of a small portion of the surface of a granule, or exposed asphalt. The corresponding micro-absorptance is $a=1-r$. It will turn out to be helpful to use the concept of thermal emittance, so we also introduce upper case $E$ and lower case $e$ for the corresponding macro- and micro- parameters. In view of Kirchhoff's law [4], $a=e$ and $A=E$. Finally, we introduce the dimensionless parameter $p$, which is a measure of surface roughness. $p$ is the probability that a photon leaving the rough surface will again encounter the surface. 


\subsection{Reflectance of a monocolor rough surface}

Consider the absorptance of a rough surface. Any incident photon will certainly encounter the surface at least once and be absorbed with probability $a$ or reflected with probability $r=1-a$. If reflected, it has probability $p$ to re-encounter the surface at which time it is again subject to absorption or reflection, and so on. We have,

$A=a+p r a+(p r)^{2} a+(p r)^{3} a+\ldots$

$A=a /(1-p r)$.

It is clear that roughness always increases the absorptance. Only if $p$ approaches zero can $A$ approach $a$.

In reality, the probability $p$ depends on the location on the rough surface. On the top of a smooth granule $p \approx 0$; down in the interstices between granules $p$ is larger. We regard $\mathrm{p}$ as the average, or typical value, characteristic of the whole surface. This approximation is similar in spirit to the mean field approximation in physics in which, for example, the dynamic electron density may be replaced simply by its mean value.

Eq. (1) can be rewritten in terms of $R$ and $r$ only as

$R=r(1-p) /(1-p r)$

Figure 2 shows $R$ as a function of $r$ for several values of $p$. Thus if a surface is rough the microreflectance $r$ must be rather high to achieve high values of $R$. From the point of view of pigment usage, this is expensive, and the need to cover a large surface area also requires additional pigment.

\subsection{Relation between probability $p$ and total shingle area}

Let $S_{1}$ be the nominal shingle area and let $S_{2}$ be the larger, full area of the rough shingle. The quantity of thermal radiation emitted by the shingle is proportional to $E S_{1}$ (times the appropriate Planck function). On the other hand, the full thermal emission of the surface, before any scattering, is proportional to $\varepsilon S_{2}$. We can write

$E S_{1}=\varepsilon S_{2}(1-p)\left[1+p r+(p r)^{2}+\ldots\right]$

$E S_{1}=\varepsilon S_{2}(1-p) /(1-p r)$.

Now, in view of Kirchhoff's law, we can replace $E$ with $A$ and $\varepsilon$ with $a$, and obtain

$A S_{1}=a S_{2}(1-p) /(1-p r)$.

This result, together with Eq. (1), proves that

$S_{2} / S_{1}=(1-p)^{-1}$. 
Thus for a flat surface, $S_{2} / S_{1}=1$, and $p=0$, whereas for a typical asphalt shingle $S_{2} / S_{1}$ $\approx 2$, and $p$ is about 0.5 .

\subsection{Shingles with several types of granules}

Most commercial shingles use blends of different granules for aesthetic reasons. Let the subscript $\mathrm{i}=1,2,3, \ldots$ label the different granule types. The numerical weighting of each granule type is $w_{\mathrm{i}}$, with $\sum_{\mathrm{i}} w_{\mathrm{i}}=1$. For example, with equal parts of two granule types, we would have $w_{1}=w_{2}=$ $1 / 2$.

The most straightforward way to estimate the reflectance of a shingle with a blend of granules is just

$R=\sum_{\mathrm{i}} w_{\mathrm{i}} R_{\mathrm{i}}$

That is, one just uses linear averaging. This approach has worked well in practice so far, as we shall see. However, we will derive small corrections to this formula that become important if the granules in question are highly reflective. The procedure is as follows.

For monocolor shingles, after measuring the shingle reflectances $R_{\mathrm{i}}$ (and neglecting the small amount of exposed asphalt), and using an estimate for $p$, we can then estimate the microreflectances $r_{\mathrm{i}}$. For a given granule blend, we then compute

$r=\sum_{\mathrm{i}} w_{\mathrm{i}} r_{\mathrm{i}}$

and $a=1-r$.

Then, Eq. (1) or (2) can be used to compute $R$ and $A$. This procedure is not the same as Eq.(4) because of the non-linear relationship between the $r$ 's and $R$ 's. For example, if we consider a $w_{1}: w_{2}$ mixture $\left(w_{2}=1-w_{1}\right)$ of granules with micro-reflectances $r_{1}$ and $r_{2}$, the extra absorptance $\triangle A$, compared to the straight linear averaging of Eq. (4) is, after some algebra,

$\Delta A=w_{1}\left(1-w_{1}\right) p(1-p)\left(r_{2}-r_{1}\right)^{2} /\left[\left(1-p r_{\mathrm{av}}\right)\left(1-p r_{1}\right)\left(1-p r_{2}\right)\right]$

where $\left.r_{\mathrm{av}}=w_{1} r_{1}+w_{2} r_{2}\right)$.

For mixtures with 3 types of granules, the extra absorptance $\Delta A$ has been computed numerically and the results are displayed in Fig. 3 (a) and (b). We can see that a kind of Murphy's law is in operation here. If one wants to design a low-absorptance (high reflectance) shingle, the straightforward linear averaging model can be rather accurate, but the extra absorption we compute as a deviation is always positive.

If we were to fabricate a cool, reflective shingle using an assortment of granules, all with reflectance in the interval $[0.18,0.36]$, the maximum possible extra absorption would occur for a 
roughly 50:50 mixture of the most- and least-reflective granules. Figure 3 (c) shows the extra absorptance using a pair of granules with mono-color shingle reflectances of 0.18 and 0.36 . The maximum extra absorptance is only about 0.007 . This example shows that for large nonlinearities to be important, not only must high reflectances be involved, but significantly different reflectances must also be involved.

\section{Reflectance of a rough shingle: granule pair model}

The granule pair model to be derived here is an alternative to the model of Sec. 2. Like our prior model, while not exact, it will nevertheless be useful for treating surface roughness. Also of value, the granule pair model specifies a definite geometry, whereas the mean field approximation treats an arbitrary surface with roughness specified by the surface area.

We consider a pair of adjacent granules labeled $\mathrm{i}$ and $\mathrm{j}$. We approximate them by a symmetrical $\mathrm{V}$ groove with micro-reflectances $r_{\mathrm{i}}$ and $r_{\mathrm{j}}$ (as before, $a_{\mathrm{i}}=1-r_{\mathrm{i}} ; a_{\mathrm{j}}=1-r_{\mathrm{j}}$ ), and assume the reflectance is highly diffuse (Lambertian). The $\mathrm{V}$ groove can be used to simulate the interreflections between the granules. Furthermore, changing the apical angle of the V can vary the effective surface roughness. Thus, for example, if the total surface area is to be twice the nominal area, the apical angle is $60^{\circ}$. Using radiant view factor algebra described in textbooks [5] on engineering heat transfer, one can show, without appealing directly to Kirchhoff's law, that $p$ again satisfies Eq. (3). However, a photon leaving surface i can only strike surface j, and vice versa. Allowing for inter-reflections, and assuming that both surfaces experience uniform light intensity, we can compute the absorptance of the $\mathrm{V}$-groove as

$A_{\mathrm{ij}}=0.5\left[\left(a_{\mathrm{i}}+a_{\mathrm{j}}\right)(1+p)-2 a_{\mathrm{i}} a_{\mathrm{j}} p\right] /\left[1-r_{\mathrm{i}} r_{\mathrm{j}} p^{2}\right]$.

While the granule pair model is different from the mean field model in general, if we assume both sides of the $\mathrm{V}$ have the same reflectance, then we do recover Eq. (1).

To compute the absorptance of a mixture of granules, we need to weight the granule pair reflectances $A_{\mathrm{ij}}$ by the probability of occurrence of the pair ij, namely $w_{\mathrm{i}} w_{\mathrm{j}}$, which leads to

$A=\sum_{\mathrm{i}} \sum_{\mathrm{j}} w_{\mathrm{i}} w_{\mathrm{j}} A_{\mathrm{ij}}$

For example, if we have a 50:50 mixture of black and white granules, we have a net weighting of 0.5 for the black-white combination and 0.25 each for the black-black and white-white combinations.

As in the prior section, we can now compute the excess absorption for a two-granule mixture, compared to the straight linear averaging approach, and find

$\Delta A=w_{1}\left(1-w_{1}\right) p(1-p)\left(r_{2}-r_{1}\right)^{2} /\left[\left(1-p^{2} r_{1} r_{2}\right)\left(1-p r_{1}\right)\left(1-p r_{2}\right)\right]$

which is very similar to, but not identical with, Eq. (6). 


\section{Shingle reflectance measurements}

\subsection{Instrumentation and measurements on mono-color shingles}

For spectral reflectance measurements, we used a Perkin-Elmer Lambda 900 spectrometer fitted with an integrating sphere. The size of the beam on the sample is only about $1 \mathrm{~mm}$ by $10 \mathrm{~mm}$ and furthermore, the width of the beam depends on the wavelength and detector in use. (We usually operate the instrument in a mode in which lower signal-to-noise ratios are compensated by widening the monochromator slit.) Since shingles have higher reflectance on the tops of granules and a lower reflectance at interstices, the measured reflectance is unduly sensitive to the exact location of the beam on the sample. Still, for mono-color shingles, the resulting spectra give a very useful representation of the spectral distribution of reflectance. Spectra are shown for 12 mono-color shingles in Figs. 4 (a) to (d). These samples were fabricated using filled asphalt substrates at a pilot plant. An idea of the magnitude of the sampling error can be seen in the artifactitious jumps in some of the spectra at $860 \mathrm{~nm}$, where the spectrometer switches detectors.

For measurements of total solar reflectance, we used a Devices \& Services Solar Spectrum Reflectometer (SSR) set for air mass 1.5. This instrument measures reflectance in 4 bands (located respectively in the ultraviolet, blue, red and near-infrared) and computes a weighted average reflectance. Diffuse illumination is used, and the detectors view the sample through a near-normal collimating tube. The aperture is $25 \mathrm{~mm}$ in diameter, with the detectors viewing the central region. Since the measurement area is larger than what is used by the spectrometer, the measurements are less sensitive to the precise location on the sample. When using the SSR instrument, measurements were furthermore obtained at a number of locations on the sample to ensure accurate estimates of the mean reflectance.

For the purposes of this paper, we regard the Devices \& Services SSR as a reference instrument. The drift in the reflectance zero is less than 0.001 . The drift while viewing the reference white tile with nominal reflectance of 0.8 is roughly 0.000 to 0.002 provided the instrument is warm and frequent calibration checks are performed. Since the reflectance of the shingle samples were all less than 0.4 , it is believed that we have reduced errors due to instrumental drift to about 0.001 .

Table 1 displays the solar reflectance values of the mono-color shingles. The measurement range shows the variation caused by the differing random positions of the measurement head on the shingle. It can be seen that the largest measurement ranges are observed on the more reflective shingles; for the least reflective shingles (e.g., black), the measurement range is small.

\subsection{Measurements on shingles with mixtures of granules}

Table 2 shows measured and estimated reflectances for 3 shingle blends. It can be seen that the linear averaging estimates are quite close to the measured values, especially for the first two blends, that resemble commercial shingle blends. The mean field model values were computed using the non-spectral (solar average) reflectances in Table 1. If a detailed spectral computation had been performed, the mean field values tabulated here would be slightly smaller. For the 50:50 mixture of black and white granules, there is a hint that the linear averaging approach may yield values that are slightly too high. In order to confirm this phenomenon we examined mixtures of black and a brighter white. 
The largest reflectance measured on the mono-color shingles at hand was 0.371 in the L2 (red) channel of the SSR instruments using the 9clwh white granules. The spectral data in Fig. 4 (a) confirm a high and not-rapidly-varying value in this spectral region. The smallest value in the L2 channel was 0.030 using the 4stdblk black granules. Mixtures of this white and black were prepared with black fractions of $0 ., 0.25,0.50,0.75$, and 1.0. The resulting data are shown in Fig. 5 , in which it is clear that a deviation from linearity has been observed. The curvature allows us to estimate a value for the probability $p$ from the data. For the mean field model, we find $p=$ 0.539 . If we use the alternative granule pair model a slightly different value $(0.604)$ is needed, but the data can be fit equally well. In passing, it is worth mentioning why we expected values near 0.5. Suppose the surface is well-covered with granules (neglect the uncovered surface), and each exposed granule is a hemisphere. Then the ratio of total to nominal area is 2 , and $p=0.5$.

\subsection{Optical effects of exposed asphalt}

Under the microscope, it is a simple matter to identify small globules of asphalt in some of the interstices between granules. The asphalt has a glossy black appearance that contrasts with the diffusely reflecting granule surfaces. It has not, however, proved simple to measure the quantity of exposed asphalt. In fact, it may be useful to turn the problem around. Rather than measure the quantity of exposed asphalt and demonstrate how the dark asphalt reduces the reflectance of otherwise reflective shingles, one can use the reflectance itself as a measure of the quality of the coverage.

One attempt to measure amount of exposed asphalt employed a flatbed scanner to measure visual brightness with a resolution of 900 dpi (dots per inch, 28 micrometer resolution). We divided one $10 \mathrm{~cm}$ x $10 \mathrm{~cm}$ sample into 9 equal sections. Then the brightness frequency distributions of the brightest and darkest sections were compared. We reasoned that the darker sections would have more exposed asphalt. Fig. 6 shows a typical measurement. The high brightness curve (with data points) exhibits fewer pixels with low reflectance in the $40-50$ region, and more pixels in the high $150-190$ region. It appears that the deviations in the $40-50$ region are indeed due to asphalt differences. However, the asphalt reflectance distribution merges smoothly with the main distribution, so that it is not obvious how to deconvolve these two distributions.

As mentioned in the introduction, our measurement of reflectances of 0.209 and 0.232 for two samples with black and white adhesive, respectively, can be used to provide a rough estimate of the effect of exposed asphalt (black adhesive). We use the simple mean field model, and treat the small amount of exposed adhesive as a second granule type with $r=0.064$ for black and $r=0.8$ for white. A rough estimate of the micro-reflectance $r$ for the 2 stdbf granule is 0.36 . From these numbers, we attribute $40 \%$ of the difference in $\Delta R$ (black vs. white adhesive) to the black vs. $r=$ 0.36 situation and the remaining $60 \%$ to the $r=0.36$ vs. white difference. Thus we estimate that the 0.209 reflectance could be raised by about 0.009 by replacing the black adhesive by a surface with the same micro-reflectance as the 2 stdbf granule.

\subsection{Texture}

The reflectances of the samples of Figs. 1 (a) and 1 (b) are 0.246 and 0.209 . Using our estimate of the effect of exposed asphalt in the prior section, the lower value here could be raised to 
0.218 . The remaining difference with the 0.246 of a well-fabricated shingle is 0.028 . (If we considered the impact of exposed asphalt on the well-fabricated shingle, the difference would be even larger.) Thus, the detailed arrangement of the granules can cause variations in the total reflectance that are more than $10 \%$ of the shingle reflectance.

Figure 6 contains further information on texture, based on 2 different sections of a wellfabricated shingle. If the only difference in these sections were amount of exposed asphalt, then both distributions would have the same shape outside the 40 - 50 brightness range. Instead, the curves are different. In particular, the high brightness section actually has fewer pixels in the highest brightness range above 200, and a disproportionally high peak at 165 . Future work is desirable to further clarify the detailed relationship between texture and reflectance.

\subsection{Size distribution}

Some variance in the size distribution is likely helpful in obtaining good substrate coverage small granules can fit in the spaces created by the larger granules. We could expect that for the same type of size distribution, but with smaller mean sizes, that the ratio of total to nominal area would be the same, and the reflectance would therefore remain the same. However, the scale of the surface roughness is only important down to dimensions on the order of the wavelength of light. Thus a smaller size distribution may reduce the effective surface area slightly, and thereby slightly increase the reflectance.

\section{Summary}

We have found that for current cool shingle design a good approach is to fabricate mono-color shingles and measure their solar reflectances. For a given shingle blend, linear averaging of the mono-color reflectances gives a good estimate of the blend reflectance.

However, more accurate methods for estimating reflectances have been derived, the mean field approach of Sec. 2 and the granule pair model of Sec. 3. The mean field approach appears more general than the granule pair model, but neither model is exact and both models yield similar results. These models account approximately for multiple scattering effects and incorporate the non-linear relationship between micro-reflectance $r$ and macro-reflectance $R$, as illustrated in Fig. 2. These more accurate models indicate that simple linear averaging slightly overestimates the reflectance of blends. Errors become larger if more reflective granules are used. Based on currently available granules and on Fig. 3 (a), we observe that the maximum possible overestimate in reflectance is 0.03 , with most recipes leading to smaller overestimates. If cool shingle design is performed with granules with macro-reflectances in the limited range of [0.18, $0.36]$, then the maximum possible overestimate in reflectance is only 0.007 .

The effect of exposed asphalt on shingle reflectance is not accurately known, but we have estimated that the asphalt-caused reduction in shingle reflectance of a well-fabricated shingle with nominal reflectance of 0.25 , is less than 0.01 . In contrast, the granule orientation as affected by manufacturing processes, may affect the reflectance by more than 0.025 , or $10 \%$ of the 0.25 nominal value. 


\section{Acknowledgments}

The work at Berkeley was supported by a grant from 3M Company to Lawrence Berkeley National Laboratory (LBNL). The work was in part supported by the California Energy Commission (CEC) through its Public Interest Energy Research Program (PIER). LBNL is operated by the University of California for the U. S. Dept. of Energy under Contract No. DEAC02-95CH11231.

\section{References}

1. Levinson, R. P. Berdahl, H. Akbari, W. Miller, I. Joedicke, J. Reilly, Y. Suzuki, M. Vondran. 2007. "Methods of Creating Solar-Reflective Nonwhite Surfaces and their Application to Residential Roofing Materials," Solar Energy Materials \& Solar Cells, 91, 304-314.

2. Levinson, R., P. Berdahl and H. Akbari. 2005. "Solar spectral optical properties of pigments, part I: Model for deriving scattering and absorption coefficients from transmittance and reflectance measurements," Solar Energy Materials \& Solar Cells, 89(4): 319-349.

3. Levinson, R., P. Berdahl and H. Akbari. 2005. "Solar spectral optical properties of pigments, part II: Survey of common colorants," Solar Energy Materials \& Solar Cells, 89(4): 351-389.

4. C. Kittel and H. Kroemer 1980, Thermal Physics, $2^{\text {nd }}$ ed., W. H. Freeman, San Francisco: 9697.

5. R. Siegel and J. Howell 2002, Thermal Radiation Heat Transfer, $4^{\text {th }}$ ed., Taylor \& Francis, New York. 


\begin{tabular}{|l|l|l|l|}
\hline Granule ID & Description & Solar reflectance & Range of measurements \\
\hline 1 stdwh & standard white & 0.286 & \pm 0.003 \\
\hline 2 stdbf & standard buff (yellowish brown) & 0.246 & \pm 0.005 \\
\hline 3 stdgr & standard gray (dark brown) & 0.082 & \pm 0.002 \\
\hline 4 stdblk & standard black & 0.033 & \pm 0.001 \\
\hline $5 \mathrm{clbr}$ & cool chestnut (brown) & 0.264 & \pm 0.006 \\
\hline $6 \mathrm{cltn}$ & cool tan (light brown) & 0.311 & \pm 0.007 \\
\hline $7 \mathrm{clbr}$ & cool sienna (orange brown) & 0.303 & \pm 0.005 \\
\hline 8clbgy & cool blue gray & 0.293 & \pm 0.006 \\
\hline 9clwh & cool (bright) white & 0.345 & \pm 0.007 \\
\hline $10 \mathrm{clblk}$ & cool black (dark brown) & 0.226 & \pm 0.004 \\
\hline $11 \mathrm{cldgy}$ & cool dark gray & 0.230 & \pm 0.005 \\
\hline $12 \mathrm{clgy}$ & cool white/gray & 0.296 & \pm 0.004 \\
\hline $13 \mathrm{clbr}$ & cool brown & 0.237 & \pm 0.005 \\
\hline $14 \mathrm{stdggy}$ & standard gray (greenish tint) & 0.132 & \pm 0.004 \\
\hline $15 \mathrm{stddbr}$ & standard dark brown & 0.069 & \pm 0.001 \\
\hline
\end{tabular}

Table 1. Reflectances of mono-color shingles (SSR, air mass 1.5). The tabulated ranges show the size of the variations in individual measurements. The reflectance values are estimates of the center of the measurement distribution.

\begin{tabular}{|l|l|l|l|l|}
\hline Blends: & $\begin{array}{l}\text { Solar } \\
\text { reflectance }\end{array}$ & $\begin{array}{l}\text { Range of } \\
\text { measurements }\end{array}$ & $\begin{array}{l}\text { Linear averaging } \\
\text { estimate }\end{array}$ & $\begin{array}{l}\text { Mean field } \\
\text { estimate }\end{array}$ \\
\hline $\begin{array}{l}\text { Weathered wood - } \\
\text { standard }\end{array}$ & 0.106 & \pm 0.006 & 0.105 & 0.102 \\
\hline $\begin{array}{l}\text { Weathered wood - } \\
\text { cool }\end{array}$ & 0.280 & \pm 0.010 & 0.286 & 0.284 \\
\hline $\begin{array}{l}\text { Black \& White } \\
\text { 50:50 } \\
\text { 4stdblk/1stdwh }\end{array}$ & 0.144 & \pm 0.010 & 0.160 & 0.145 \\
\hline
\end{tabular}

Table 2. Measured solar reflectances of 3 blends. The first two are representative of commercial standard and cool blends. The third blend is a 50:50 mixture of white and black that does not resemble a commercial shingle, but which is chosen to seek maximum deviation from the linear averaging estimate. The standard weathered wood recipe (fractions and granule codes) is 0.10 , 15 stddbr; $0.65,3$ stdgy; $0.15,14$ stdggy; and $0.10,2$ stdbf. The cool weathered wood recipe is 0.05, 10clblk; 0.05, 11 cldgy; 0.20, 13clbr; 0.15, 8clbgy; 0.20, 12clgy; 0.25, 6cltn; and 0.10, 9 clwh. For the mean field estimates $p=0.539$, based on Fig. 5. 


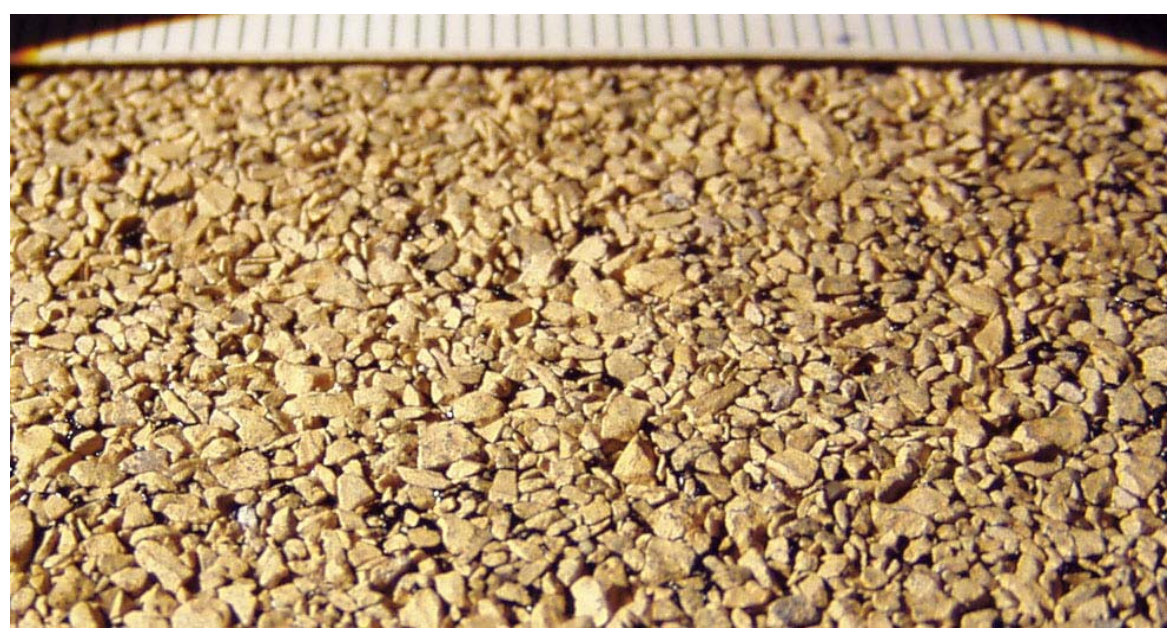

(a)

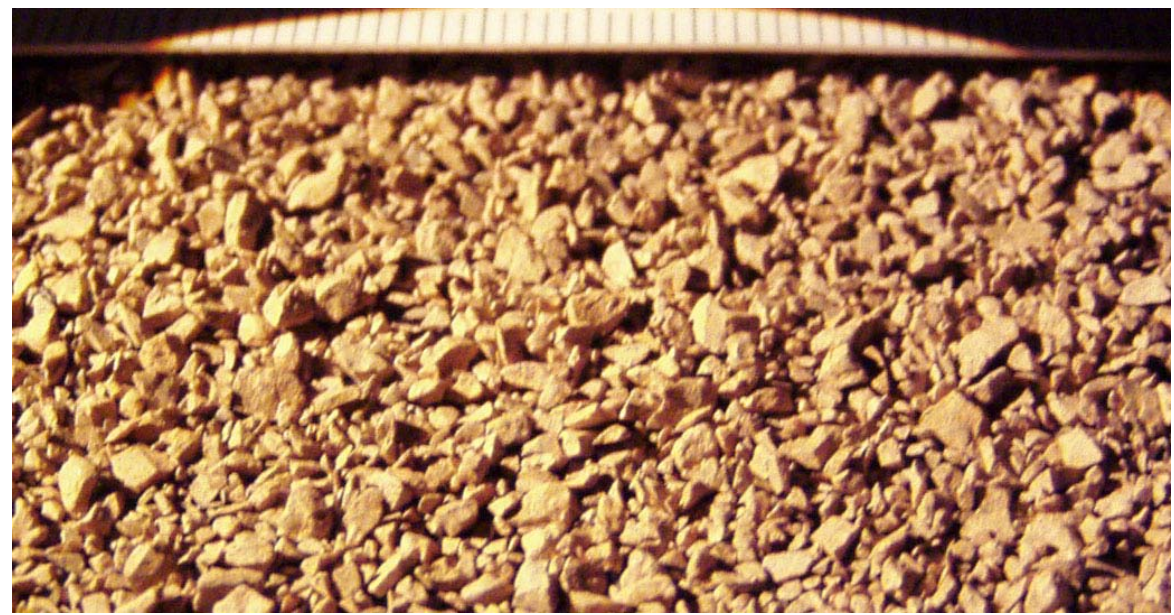

(b)

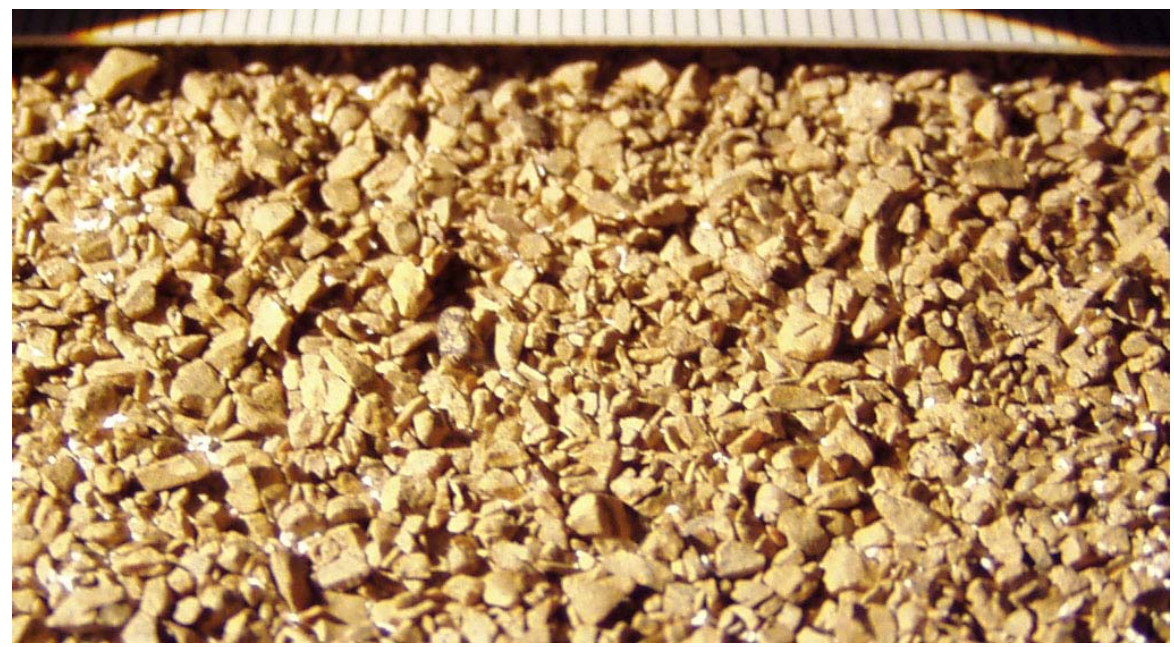

(c)

Fig. 1. Photographs of tested shingles with 2 stdbf granules (see Table 1): (a) pilot plant shingle, (b) hand-made shingle with black paint as adhesive, and (c) hand-made shingle using white paint as adhesive. The ruler shows millimeters. 


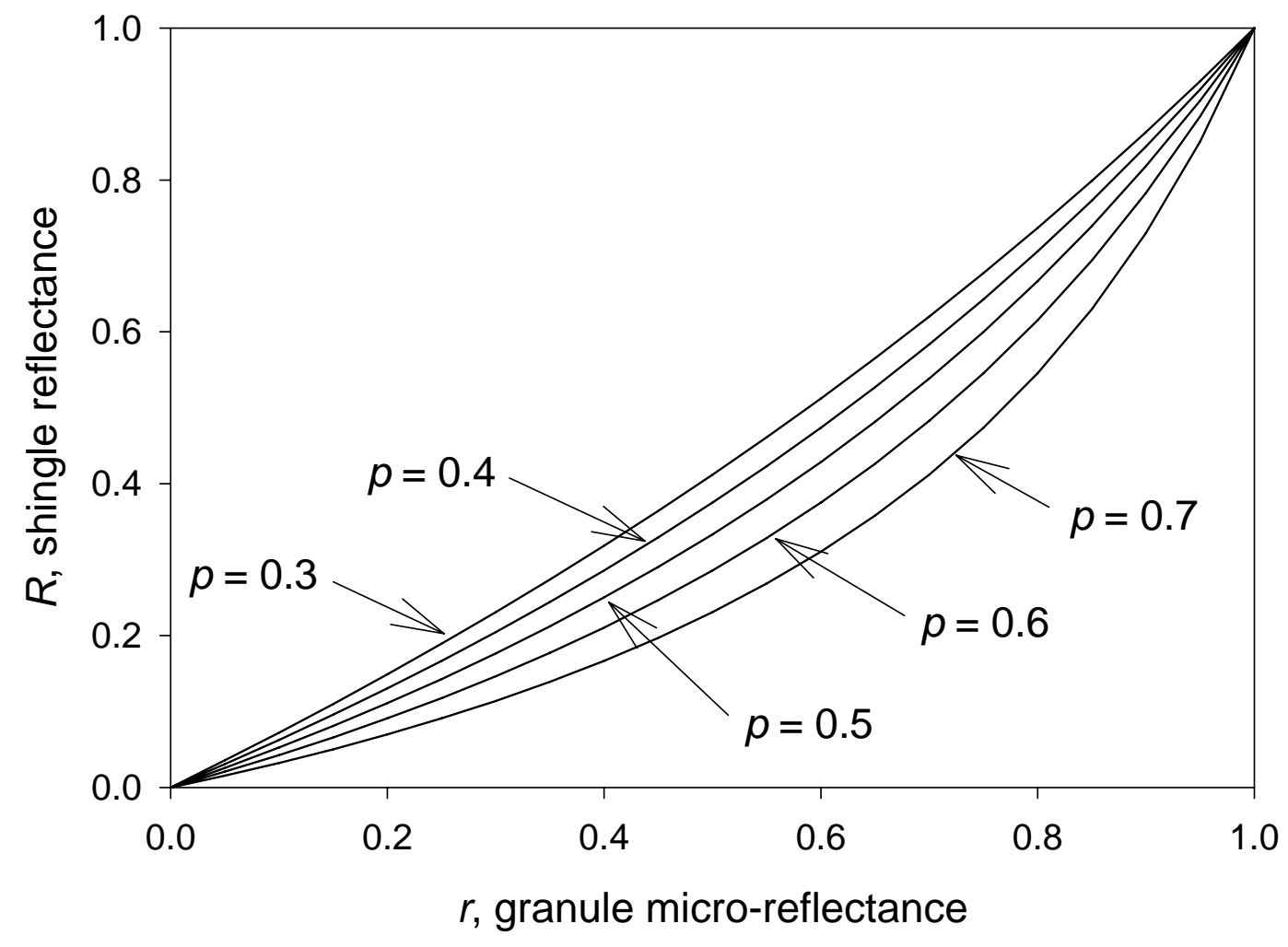

Fig. 2. The relation between shingle macro-reflectance and granule surface micro-reflectance for several values of $p$. The ratio of total shingle area to nominal area is approximately $(1-p)^{-1}$. 


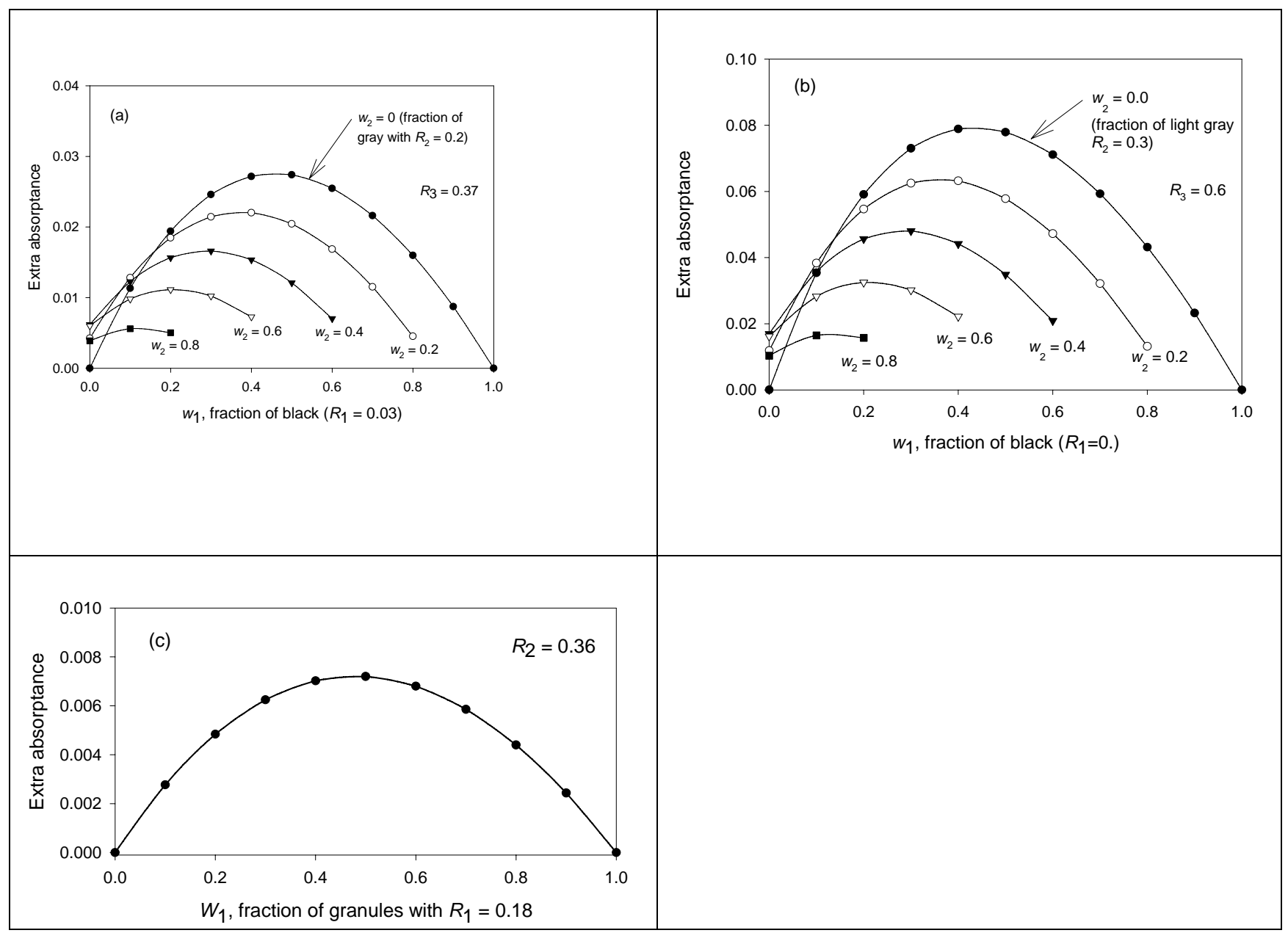

Fig. 3.. These plots show the extra absorptance, compared to simple linear averaging of Eq. (4), as computed based on Eq. (5). Compositions using up to 3 granule types labeled 1, 2, and 3 are included. $w_{3}=1-w_{1}-w_{2}$. (a) The highest reflectance of $R_{3}=0.37$ is the highest measured value for a white granule, obtained in channel L2 (red spectral region) of the Solar Spectrum Reflectometer as discussed in the text. $R_{1}=0.03$, the lowest value, for a black granule. The assumed value of $R_{2}=0.2$ corresponds to a medium value, say gray. The largest value of $\triangle A$ is about 0.027 , obtained with $w_{2}=0$ (no gray) and an approximately 50:50 mixture of black and white. (b) Plot as in (a), but with still more reflective granules. Here $R_{3}=$ bright white $=0.6, R_{2}=$ light gray $=0.3$, and $R_{1}=$ jet black $=0.0$. In this case, errors as large as 0.08 can occur if Eq. (4) is used instead of Eq. (5). (c) Extra absorption for a two-granule mixture with reflectances of 0.18 and 0.36 . The maximum value is about 0.007 , which is therefore also the maximum excess absorption for any number of granules all with mono-color shingle reflectances in the range $[0.18,0.36]$. 


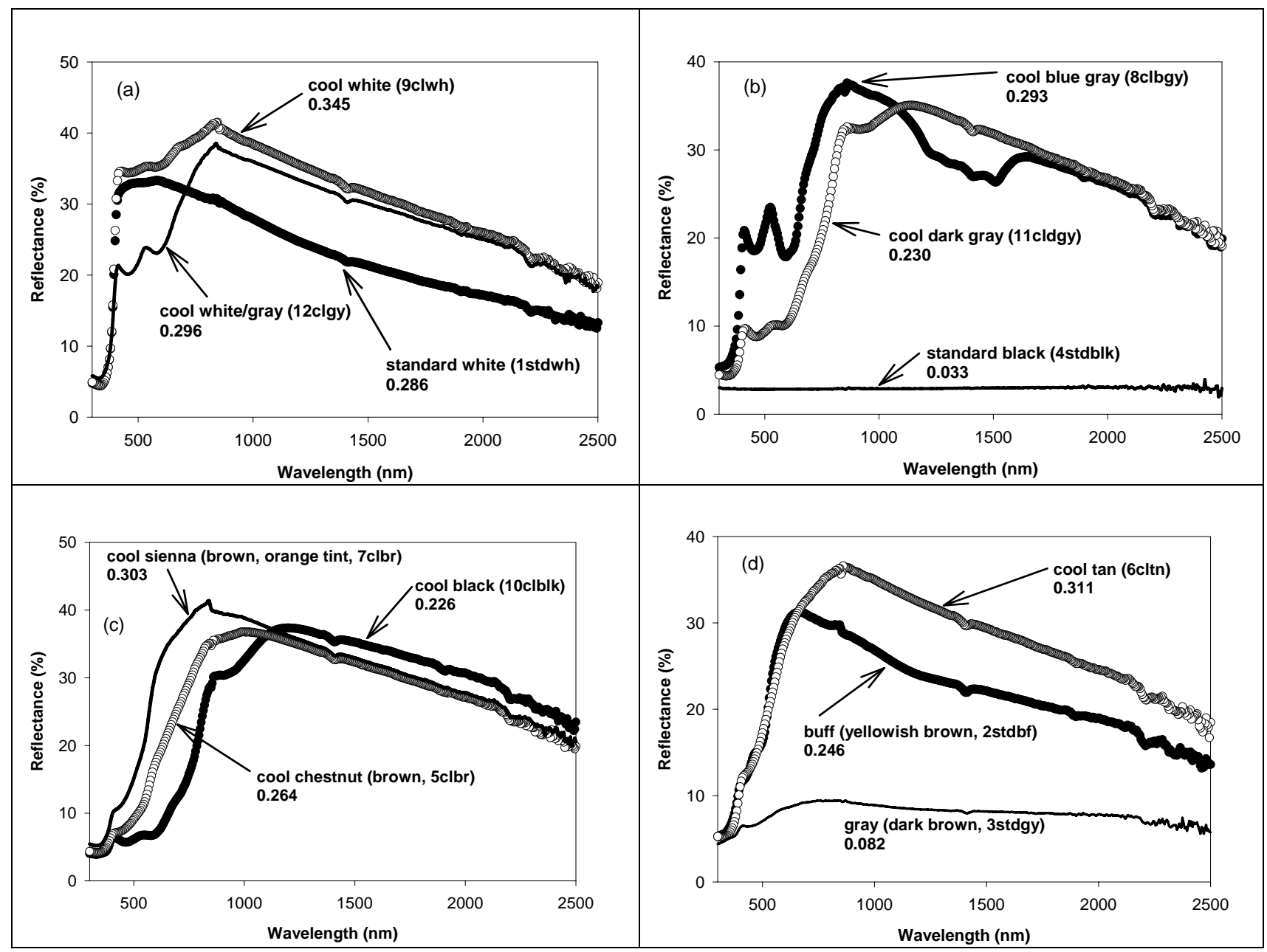

Fig 4. Spectral reflectance of (a) 3 white shingles (b) gray and black shingles, (c) brown and black shingles, and (d) 3 more brown shingles. Granule labels are from Table 1. SSR reflectances are listed below the labels. 
Mixtures of 9clwh white and 4stdblk black granules
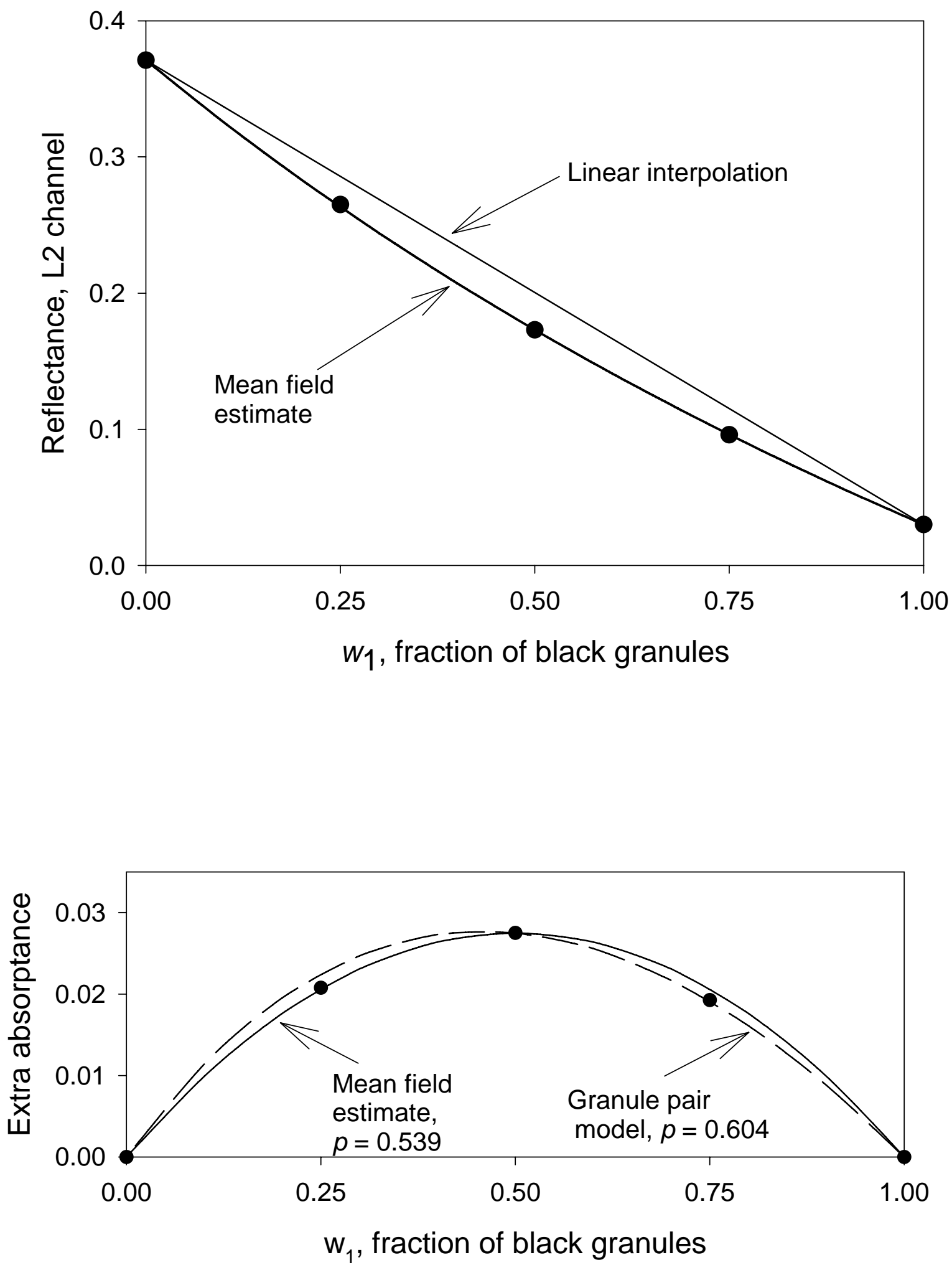
Fig. 5. To fit the reflectance as a function of $w_{1}$, the fraction of black granules, the probability $p$ is used as a free parameter. The detail in the lower panel shows that both the mean field and granule pair models can be made to fit the data well, but require slightly different values for $p$.

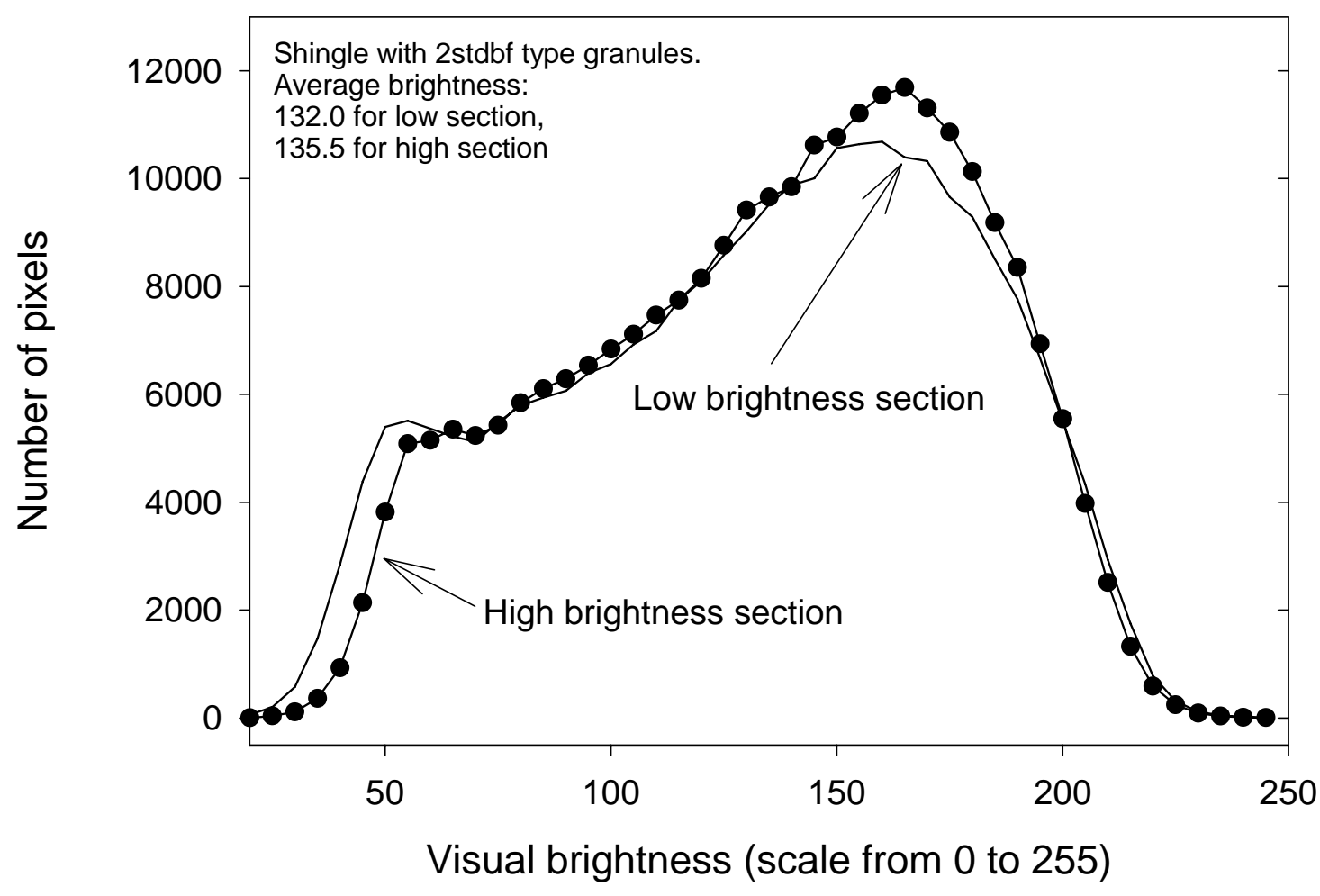

Fig. 6. Frequency distribution of brightness for both high and low brightness sections of monocolor shingle with 2 stdbf granules. 